\title{
DEFORMATION POTENTIAL LIMITED SPIN RELAXATION IN CUBIC SEMICONDUCTORS*
}

\author{
A. Dargys \\ Semiconductor Physics Institute, A. Goštauto 11, LT-01108 Vilnius, Lithuania \\ E-mail: dargys@pfi.lt
}

Received 13 April 2005

\begin{abstract}
The scattering of valence band hole spin by acoustical and optical phonons due to deformation potential interaction is considered. Six-band deformation potential matrix, which takes into account doubly degenerate heavy-mass, light-mass, and split-off energy bands, is used to evaluate the matrix elements for spin conserving and flipping transitions. The concept of the spin surface was addressed to define the initial and final states of the hole spin. It was found that, in agreement with experiment, the spin conserving and flipping transitions are of comparable magnitude in both intravalence and intervalence band scattering mediated by acoustical as well as optical phonons.
\end{abstract}

Keywords: spintronics, spin relaxation, spin dephasing, hole spin, deformation potential scattering

PACS: 85.75.-d, 72.25.Rb, 67.57.Lm

\section{Introduction}

Due to enormous potentiality of applications of the spin-based or so-called "spintronic" devices, intense experimental and theoretical efforts are concentrated on the physics of electron spin in semiconductors and metals best reflected in the recent review by Žutić et al. [1]. However, to realize a useful spintronic device the spin memory of the device should be long, or at least longer than an elementary working cycle of the device. Therefore, the understanding of physics of spin relaxation mechanisms and ways to control relaxation is of the paramount importance in the development of any spintronic device.

A large class of spintronic devices (spin transistors, spin filters, spin LEDs) are based on a coherent spin transport through channels bridged by ferromagnetic contacts and/or quantum wells. There are three basic spin relaxation mechanisms for free electrons and holes in 2D channels as well as in the bulk of these devices suggested some time ago by Elliott and Yafet (EY) [2,3], D'yakonov and Perel (DP) [4], and Bir, Aronov, and Pikus (BAP) [5]. At present, the importance of the proposed mechanisms is confirmed experimentally [1].

\footnotetext{
* The report presented at the 36th Lithuanian National Physics Conference, 16-18 June 2005, Vilnius, Lithuania.
}

In the EY mechanism the flipping of charge carrier spin is related with the spin-orbit (SO) interaction. Here the spin flipping comes from one of the spinors (initial or final) which is expanded to a higher order in the scattering matrix to include SO interaction, while the other (final or initial) spinor may not necessarily include the SO interaction. Thus, in EY mechanism the wave functions take into account the SO interaction, while the interaction operator does not necessarily includes it. In the DP mechanism the spin lifetime is related with motional spectral line narrowing. In noncentrosymmetric semiconductors the conduction band is spin-split. The beating between the two closely spaced spin-split conduction bands gives rise to a slow precession of the spin polarization. However, the electron collisions with lattice phonons change the direction of spin precession axis randomly. If electron-phonon collisions are fast in comparison with an average precession period, the spin lifetime becomes long due to motional line narrowing effect [6], where a compensation of the wave function dephasing between random scattering events takes place. The DP mechanism is absent in diamond-type semiconductors, where bands remain doubly degenerate at all electron energies. In the BAP mechanism, the spin flipping comes from exchange interaction between the spins of free electrons and holes, or acceptors during collisions. Usually the last of the mechanisms is effective only at large doping levels. 
Recently Song an Kim [7] have reviewed all three spin relaxation mechanisms and have constructed a kind of phase diagrams where they have depicted graphically which mechanism is dominant in each portion of semiconductor parameter space.

Up to present, most attention was paid to the conduction band electron spin relaxation since the electron spin lifetime is comparatively long. In the case of valence bands the situation is different. Here the SO interaction is strong enough to transform the valence band structure and spin properties radically. As a result, the spin is no longer a good quantum number in transition matrix elements and the meaning of "spin conserving" and "spin flipping" transitions requires a more critical analysis. Due to rearrangement of the bands, the hole spin may experience strong scattering after a single momentum randomizing collision, as the experiments show [8-10].
In this article, a new approach based on a concept of spin surface [11-13] is used to calculate spin conserving and flipping matrix elements of the hole under deformation potential interaction. The spin surface represents all possible spin directions and lengths of polarization vector of the hole that propagates ballistically in either heavy-mass, light-mass or split-off band. The parametrization of the spin surface circumvents the complicated procedure of constructing the six-band hole spinors from the semiconductor constants. The knowledge of the spin conserving and flipping matrix elements allows one to easily obtain the spin relaxation time from the momentum relaxation time. The latter has been thoroughly investigated in $p$-type semiconductors [14-17]. The interaction considered here is of EY-type [2,3].

\section{Deformation potential matrices. Initial and final spinors}

The scattering of spin will be considered in a model of doubly degenerate heavy-mass, light-mass, and split-off bands that are typical to elementary semiconductors. To calculate the spin-dependent intra- and intervalence transition probability one must know the deformation potential matrices. For parabolic and degenerate four-band (heavyand light-mass) model, such deformation potential matrices for the acoustic and optical phonons can be found in the monograph by Bir and Pikus [18]. These matrices can be generalized for six band optical [19] and acoustical [20] deformation potentials, where spin-orbit split-off band is included. In the total angular momentum basis $|J, m\rangle$, which will be written in the order $\left.\left(\left|\frac{3}{2}, \frac{3}{2}\right\rangle,\left|\frac{3}{2}, \frac{1}{2}\right\rangle,\left|\frac{3}{2},-\frac{1}{2}\right\rangle,\left|\frac{3}{2},-\frac{3}{2}\right\rangle,\left|\frac{1}{2}, \frac{1}{2}\right\rangle, \frac{1}{2},-\frac{1}{2}\right\rangle\right)$, both the acoustical and optical deformation potential matrices have the same structure:

$$
H_{\mathrm{DP}}^{a c, o p}=\left[\begin{array}{cccccc}
f & h & j & 0 & -\frac{\mathrm{i}}{\sqrt{2}} h & \mathrm{i} \sqrt{2} j \\
h^{*} & g & 0 & j & \frac{\mathrm{i}}{\sqrt{2}}(f-g) & -\mathrm{i} \sqrt{\frac{3}{2}} h \\
j^{*} & 0 & g & -h & \mathrm{i} \sqrt{\frac{3}{2}} h^{*} & \frac{\mathrm{i}}{\sqrt{2}}(f-g) \\
0 & j^{*} & -h^{*} & f & \mathrm{i} \sqrt{2} j^{*} & \frac{\mathrm{i}}{\sqrt{2}} h^{*} \\
\frac{\mathrm{i}}{\sqrt{2}} h^{*}-\frac{\mathrm{i}}{\sqrt{2}}(f-g) & -\mathrm{i} \sqrt{\frac{3}{2}} h & -\mathrm{i} \sqrt{2} j & \frac{1}{2}(f+g) & 0 \\
-\mathrm{i} \sqrt{2} j^{*} & \mathrm{i} \sqrt{\frac{3}{2}} h^{*} & -\frac{\mathrm{i}}{\sqrt{2}}(f-g) & -\frac{\mathrm{i}}{\sqrt{2}} h & 0 & \frac{1}{2}(f+g)
\end{array}\right] .
$$

In case of acoustical deformation potential, the elements of the matrix (1) are expressed through three deformation potential constants $l, m$, and $n$, and the strain tensor components $\varepsilon_{i j}$ in the following way:

$$
f=\frac{l+m}{2}\left(\varepsilon_{x x}+\varepsilon_{y y}\right)+m \varepsilon_{z z},
$$

$$
\begin{aligned}
& g=\frac{1}{3}\left(f+2 m\left(\varepsilon_{x x}+\varepsilon_{y y}\right)+2 l \varepsilon_{z z}\right), \\
& h=\frac{n}{\sqrt{3}}\left(\mathrm{i} \varepsilon_{x z}-\varepsilon_{y z}\right),
\end{aligned}
$$




$$
j=\frac{1}{\sqrt{3}}\left(\frac{l-m}{2}\left(\varepsilon_{x x}-\varepsilon_{y y}\right)+\mathrm{i} n \varepsilon_{x y}\right) .
$$

In practice, a different set of the deformation potential constants is frequently used: $a=(l+2 m) / 3$, $b=(l-m) / 3, d=n / \sqrt{3}$. In the continuum limit, the strain tensor $\varepsilon_{i j}$ is proportional to acoustic wave polarization vector $\mathbf{p}$ and wave vector $\mathbf{q}$ components: $\varepsilon_{i j} \propto 2\left(p_{j} q_{i}+p_{i} q_{j}\right)$. For longitudinal acoustic (LA) phonons the vectors $\mathbf{p}$ and $\mathbf{q}$ are parallel and only diagonal components matter. Then the strain tensor is diagonal,

$$
\varepsilon_{i j}(\mathrm{LA})=\left(\begin{array}{ccc}
q_{x} & 0 & 0 \\
0 & q_{y} & 0 \\
0 & 0 & q_{z}
\end{array}\right)
$$

For transverse acoustic wave polarizations TA1 and TA2, the vectors $\mathbf{p}$ (TA1) and $\mathbf{p}$ (TA2) are perpendicular to $\mathbf{q}$ and at the same time they are mutually perpendicular. These properties yield the following strain tensors [18]:

$$
\begin{aligned}
& \varepsilon_{i j}(\mathrm{TA} 1)=\frac{1}{\sqrt{q_{x}^{2}+q_{y}^{2}}} \\
& \times\left(\begin{array}{ccc}
0 & -q_{x}^{2}+q_{y}^{2} & q_{y} q_{z} \\
-q_{x}^{2}+q_{y}^{2} & 0 & -q_{x} q_{z} \\
q_{y} q_{z} & -q_{x} q_{z} & 0
\end{array}\right) \\
& \varepsilon_{i j}(\mathrm{TA} 2)=\frac{1}{q \sqrt{q_{x}^{2}+q_{y}^{2}}} \\
& \times\left(\begin{array}{ccc}
0 & 2 q_{x} q_{y} q_{z} & -q_{x}\left(q^{2}-2 q_{z}^{2}\right) \\
2 q_{x} q_{y} q_{z} & 0 & -q_{y}\left(q^{2}-2 q_{z}^{2}\right) \\
-q_{x}\left(q^{2}-2 q_{z}^{2}\right) & -q_{y}\left(q^{2}-2 q_{z}^{2}\right) & 0
\end{array}\right)
\end{aligned}
$$

The momentum and energy conservation in the holephonon collisions was used to eliminate phonon wave vector $\mathbf{q}$ in the favour of the vector $\mathbf{k}$. The collisions were assumed to be elastic.

In the case of optical deformation potential the matrix elements in (1) are

$$
\begin{aligned}
& f=0, \\
& g=0,
\end{aligned}
$$

$$
\begin{aligned}
& h=d_{0}\left(\mathrm{i} \delta_{\lambda y}+\delta_{\lambda x}\right), \\
& j=\mathrm{i} d_{0} \delta_{\lambda z},
\end{aligned}
$$

where $d_{0}$ is the optical deformation potential constant, and $\mathrm{i}=\sqrt{-1}$. In (6), $\boldsymbol{\delta}_{\lambda}=\left(\delta_{\lambda x}, \delta_{\lambda y}, \delta_{\lambda z}\right)$ is the direction of the displacement of atoms in an elementary cell, and $\lambda$ characterizes the phonon mode (LO, TO1, TO2). In the tetrahedral semiconductors, in small wave vector limit $(\mathbf{q} \approx 0)$ the cubic axes were chosen as arbitrary polarization directions of three orthogonal optical phonon modes: $\boldsymbol{\delta}_{\mathrm{TO} 1}=(1,0,0), \boldsymbol{\delta}_{\mathrm{TO} 2}=(0,1,0)$, $\boldsymbol{\delta}_{\mathrm{LO}}=(0,0,1)$.

The next point to consider is the selection of initial and final spinors, the product of which with the deformation potential matrix yields the transition probability. Due to large SO contribution in the formation of the valence band, the main difficulty here comes from noncommutivity of the valence band Hamiltonian and the spin operator. This means that the hole energy and spin cannot be good quantum numbers simultaneously. As shown earlier [11-13], the ballistic hole with a given wave vector $\mathbf{k}$ and energy $E_{j}(\mathbf{k})$, where $j$ is the band index, can be properly described if one, in addition, introduces the spin surface that characterizes all possible hole spin polarizations. For example, the spin surface of the conduction band electron is represented by a sphere, or is very close to a sphere, since the SO interaction has very weak influence on the electron wave function. However, in case of holes as shown in [1113], the deviation from sphere may be very large. In particular, in tetrahedral and III-V semiconductors the heavy-hole spin surface is cigar-shaped (for parabolic bands it shrinks to a line) with rotation axis parallel to k. The light-hole spin surface appears to be deformed in the opposite direction, i. e., it has a form of an oblate spheroid. The shape of the spin surface can be determined by parametrizing the spinor that represents the hole with a well-defined $\mathbf{k}$ and $E_{j}(\mathbf{k})$. For example, in the energy representation one of the possible schemes of parametrization of the heavy-hole spinor may be the following: $|f\rangle_{\mathrm{H}}=\left(0,0, \cos \vartheta, \sin \vartheta \mathrm{e}^{\mathrm{i} \phi}, 0,0\right)$, where the parameters $\vartheta$ and $\phi$ control the magnitude and direction of the spin polarization vector. In writing down the spinor in energy representation it has been supposed that the energy band order in the spinor is (L,L,H,H,S,S). The dispersion laws of spherical heavymass hole $(\mathrm{H})$, light-mass hole (L), and spin-orbit splitoff hole (S) have been described by the following dispersion laws:

$$
E_{\mathrm{H}}=\left(\gamma_{1}-2 \gamma_{2}\right) k^{2} / 2,
$$




$$
\begin{aligned}
& E_{\mathrm{L}}=\left(\gamma_{1}+2 \gamma_{2}\right) k^{2} / 2-2 \Delta \lambda^{2}, \\
& E_{\mathrm{S}}=\gamma_{1} k^{2} / 2+\Delta+2 \Delta \lambda^{2},
\end{aligned}
$$

where $k$ is the modulus of the hole wave vector, $\gamma_{1}$ and $\gamma_{2}$ are the valence band parameters, $\Delta$ is the spin-orbit splitting energy, and $\lambda$ is the nonparabolicity parameter, $\lambda=k^{2} \gamma_{2} / \Delta$. In the numerical calculations the parameters of GaAs have been used: $\gamma_{1}=6.85, \gamma_{2}=2.1$, $\Delta=0.341 \mathrm{eV}$.

If one wants to return back to the initial representation, $|\psi\rangle_{\mathrm{H}, \mathrm{L}, \mathrm{S}}=T^{\dagger}|f\rangle_{\mathrm{H}, \mathrm{L}, \mathrm{S}}$, one must know the unitary transformation matrix $T$ that connects the energy and initial (Luttinger-Kohn) representations. The matrix $T$ can be represented analytically if constant energy surfaces are spherical $[13,19]$.

\section{Spin scattering matrix}

Collision of the hole with a phonon changes the hole wave vector from $\mathbf{k}$ to $\mathbf{k}^{\prime}$. The scattering may occur within the same (intravalence scattering) or between different, $i$ and $i^{\prime}$, bands (intervalence scattering). The strength of the scattering for a selected phonon type $\alpha=\mathrm{LA}, \alpha=\mathrm{LO}$, TA1, TO1, or TA2, TO2 is described by the scattering matrix

$$
\begin{aligned}
& M_{i^{\prime} i}^{\alpha}= \\
& =\left[\begin{array}{ll}
\left|\left\langle i^{\prime} \mathbf{k}^{\prime} \uparrow^{\prime}\left|H_{\mathrm{DP}}^{\alpha}\right| i \mathbf{k} \uparrow\right\rangle\right|^{2} & \left|\left\langle i^{\prime} \mathbf{k}^{\prime} \uparrow^{\prime}\left|H_{\mathrm{DP}}^{\alpha}\right| i \mathbf{k} \downarrow\right\rangle\right|^{2} \\
\left|\left\langle i^{\prime} \mathbf{k}^{\prime} \downarrow^{\prime}\left|H_{\mathrm{DP}}^{\alpha}\right| i \mathbf{k} \uparrow\right\rangle\right|^{2} & \left|\left\langle i^{\prime} \mathbf{k}^{\prime} \downarrow^{\prime}\left|H_{\mathrm{DP}}^{\alpha}\right| i \mathbf{k} \downarrow\right\rangle\right|^{2}
\end{array}\right] .
\end{aligned}
$$

The initial and final spin polarizations, $(\uparrow, \downarrow)$ and $\left(\uparrow^{\prime}\right.$, $\downarrow^{\prime}$ ), respectively, are measured with respect to some arbitrarily selected quantization axes, where the up and down arrows indicate hole spins aligned or opposed to the axes. The directions of the initial and final quantization axes, in general, may be different. In the following it is assumed that the initial and final axis are parallel, respectively, to $\mathbf{k}$ and $\mathbf{k}^{\prime}$. The scattering will be called "spin conserving" and "spin flipping" if the final spin is along or opposed to the final quantization axes. The sum of all matrix elements in the scattering matrix (10) gives the standard scattering probability from $(i, \mathbf{k})$ to $\left(i^{\prime}, \mathbf{k}^{\prime}\right)$ state, which is usually used in the hole transport theory [15-18].

The selection of the initial and final spin polarization vector $\langle\mathbf{S}\rangle$ was done in the following way. At first, the spin surfaces were precalculated for some assumed initial/final k's and bands $i=\mathrm{H}$, L, or $\mathrm{S}$, and then in the parametrized spinors the parameters $\vartheta$ and $\phi$ were selected so that the vector $\langle\mathbf{S}\rangle$, the end of which lies on the spin surface, was pointing along or against the assumed quantization axis. For example, it was found that for spherical heavy-mass band the quantization axis and $\mathbf{k}$ were aligned in the same direction when $\vartheta=\pi / 2-\theta / 2, \phi=\pi / 2+\varphi$ and in the opposite directions when $\vartheta=\theta / 2, \phi=-\pi / 2+\varphi$, with $\theta$ and $\phi$ being the polar angles of the wave vector. In a more general case, when $\mathbf{k}$ and the quantization axis were not colinear, $\vartheta$ and $\phi$ were precalculated numerically. Since the optical phonon limited deformation potential matrix is simpler, the optical scattering will be considered first.

\section{Optical phonon limited spin relaxation}

Calculations have shown that the probabilities of spin flipping and conserving transitions in all cases, i. e., for all possible phonon modes $\alpha$, bands $\left(i, i^{\prime}\right)$, initial $(\uparrow, \downarrow)$ and final $\left(\uparrow^{\prime}, \downarrow^{\prime}\right)$ spin directions, are equal when the quantization axis is parallel to the wave vector. For example, for heavy-mass-band intravalence scattering the equation (10) gives

$$
\begin{aligned}
& M_{\mathrm{HH}}^{\mathrm{LO}}=\frac{3}{4} d_{0}^{2} \sin ^{2} \theta\left[\begin{array}{ll}
\sin ^{2} \frac{\theta}{2} & \cos ^{2} \frac{\theta}{2} \\
\cos ^{2} \frac{\theta}{2} & \sin ^{2} \frac{\theta}{2}
\end{array}\right], \\
& M_{\mathrm{HH}}^{\mathrm{TO}}=\frac{3}{2} d_{0}^{2} \sin ^{2} \theta\left[\begin{array}{ll}
\cos ^{2} \frac{\theta}{2} & \sin ^{2} \frac{\theta}{2} \\
\sin ^{2} \frac{\theta}{2} & \cos ^{2} \frac{\theta}{2}
\end{array}\right],
\end{aligned}
$$

where superscript TO indicates the sum over two transverse optical phonon modes $(\mathrm{TO}=\mathrm{TO} 1+\mathrm{TO} 2)$, and $\theta$ is the angle between the initial and final wave vectors. Matrices (11) and (12) are independent of the small parameter $\lambda$ up to the second order. The sum over all initial and final spins gives the well-known intraband scattering dependence on the scattering angle which is proportional to $\sin ^{2} \theta$. Similar matrix has been obtained for intravalence light-mass band scattering, however, the scattering strength now depends on band nonparabolicity: $M_{\mathrm{LL}}^{\mathrm{LO}}=(1+2 \lambda)^{2} M_{\mathrm{HH}}^{\mathrm{LO}}$ and $M_{\mathrm{LL}}^{\mathrm{TO}}=\left(1-2 \lambda+4 \lambda^{2}\right) M_{\mathrm{LL}}^{\mathrm{TO}}$. 
The scattering within the split-off band has been found to be proportional to $\lambda^{2}$. For example, for LO phonons it is

$$
M_{\mathrm{SS}}^{\mathrm{LO}}=3 \lambda^{2} d_{0}^{2} \sin ^{2} \theta\left[\begin{array}{ll}
\sin ^{2} \frac{\theta}{2} & \cos ^{2} \frac{\theta}{2} \\
\cos ^{2} \frac{\theta}{2} & \sin ^{2} \frac{\theta}{2}
\end{array}\right] .
$$

At $\lambda=0$ (parabolic bands) the absence of optical deformation-potential scattering in the split-off band follows from the symmetry considerations, too.

In case of the intervalence scatterings, the most important are transitions between heavy- and light-hole bands. The respective matrix elements are

$$
\begin{aligned}
M_{\mathrm{HL}}^{\mathrm{LO}}= & \left(1+4 \lambda+6 \lambda^{2}\right) d_{0}^{2}\left[\begin{array}{rr}
\sin ^{6} \frac{\theta}{2} & \cos ^{6} \frac{\theta}{2} \\
\cos ^{6} \frac{\theta}{2} & \sin ^{6} \frac{\theta}{2}
\end{array}\right], \\
M_{\mathrm{HL}}^{\mathrm{TO}}= & \frac{1}{4} d_{0}^{2} \\
& \times\left[\begin{array}{ll}
\left(F_{1}+F_{2}\right) \cos ^{2} \frac{\theta}{2} & \left(F_{1}-F_{2}\right) \sin ^{2} \frac{\theta}{2} \\
\left(F_{1}-F_{2}\right) \sin ^{2} \frac{\theta}{2} & \left(F_{1}+F_{2}\right) \cos ^{2} \frac{\theta}{2}
\end{array}\right],
\end{aligned}
$$

where $F_{1}=\left(1-2 \lambda+6 \lambda^{2}\right)(3+\cos 2 \theta)$ and $F_{2}=$ $\left(4-8 \lambda-48 \lambda^{2}\right) \cos \theta$.

To compare the strengths of the various mechanisms, the matrix elements averaged over scattering angle $\theta$ and summed over LO, TO1, TO2 phonons have been calculated. For example, equations (11) and (12) give the following averaged matrix:

$$
\left\langle M_{\mathrm{HH}}\right\rangle=\frac{3}{4} d_{0}^{2}\left[\begin{array}{ll}
1 & 1 \\
1 & 1
\end{array}\right] .
$$

In all cases, when the quantization axis is parallel to $\mathbf{k}^{\prime}$, it has been found that scattering matrices averaged over $\theta$ and summed over phonon modes reduce to a matrix similar to (16) that consists of the product of 1's and the scalar that is proportional to the matrix element for hole momentum scattering. Such structure shows that the conserving and flipping scattering probabilities are equal. From comparison of various averages it has been found that the ratio of the squares of the matrix elements for various elementary processes, when $\lambda \approx$ 0 , is $(\mathrm{SH}, \mathrm{SL}):(\mathrm{HH}, \mathrm{LL}, \mathrm{LH}):(\mathrm{SS})=1: \frac{1}{2}: 0$.

\section{Acoustical phonon limited spin relaxation}

For acoustical phonon scattering the deformation potential matrix is more involved and, as a result, the matrix elements have been found to be rather complicated. Here numerical results for some typical situations will be presented mainly in a form of graphs.

In Fig. 1 the dependence of the square of matrix element is calculated at various directions of the final wave vector given by spherical angles $\theta$ and $\phi$. The initial wave vector was $\mathbf{k}=\left(0,0, k_{z}\right)$. The collisions were assumed to be elastic and the relation $q=2 k \sin (\theta / 2)$ between the phonon $q$ and final hole wave vector $k$ was used to eliminate $q$. In the isotropic scattering case $(b=d=0)$ the matrix elements for LA phononlimited scattering reduces to

$$
M_{\mathrm{HH}}^{\mathrm{LA}}=\frac{1}{2} a^{2} q^{2}\left[\begin{array}{ll}
f^{(+)} & f^{(-)} \\
f^{(-)} & f^{(+)}
\end{array}\right],
$$

where $f^{( \pm)}=\left(4-3 \sin ^{2} \theta \pm \sin ^{3} \theta\right)$. The respective matrix for TA phonons is zero in this approximation: $M_{\mathrm{HH}}^{\mathrm{TA}}=0$. The sum of all matrix elements in (17) gives the well-known [18] momentum scattering matrix element proportional to $2 a^{2} q^{2}\left(1+3 \cos ^{2} \theta\right)$. Figure 1 shows that the spin "flipping" and "conserving" transitions have a different character. Also, the figure shows that the spin "flipping" scattering is by an order of magnitude larger then the spin "conserving" transition. From Fig. 1(b) it follows that in case of the spin flipping the scattered hole spin tends to be antiparallel to the final wave vector $\mathbf{k}^{\prime}$ direction. Thus, the acoustic phonon scattering is strongly anisotropic, and the isotropic approximation used in the literature does not reflect a real situation. Since the band dispersion is assumed spherical, it is clear that the anisotropy comes from the deformation potential matrix, where the following deformation potential constants $-a=3.1 \mathrm{eV}$, $b=-1.7 \mathrm{eV}, d=-4.55 \mathrm{eV}-$ have been used for GaAs. These constants take into account the cubic symmetry of the crystal.

Figure 2 shows the squares of the matrix elements for intervalence scattering between heavy- and lightmass bands under similar conditions. Here the spin "flipping" scattering is stronger, too.

As in the previous section, to compare between the strengths of various transitions we have integrated the matrix elements over $\theta$ and $\varphi$ and summed over LA, TA1, and TA2 phonons. The following ratios of "conserving" and "flipping" transitions $(+k /-k)_{i i^{\prime}}$, where $\pm k$ indicates that the final hole spin is either aligned or 


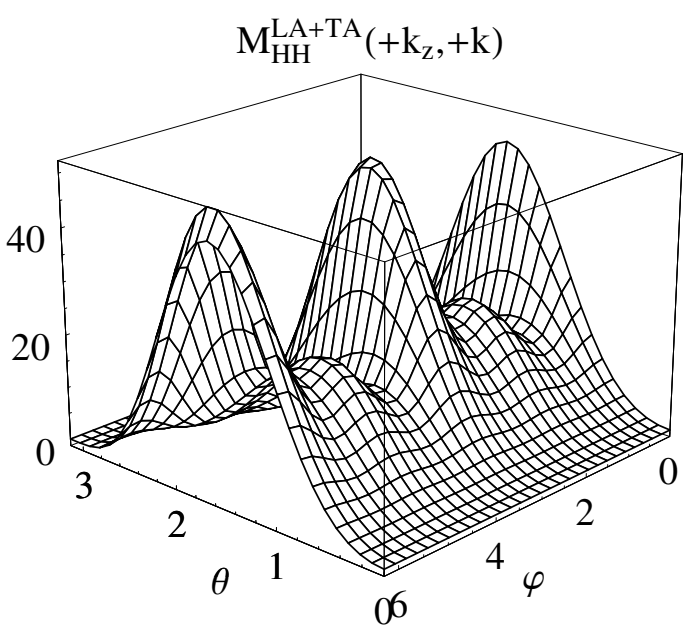

(a)

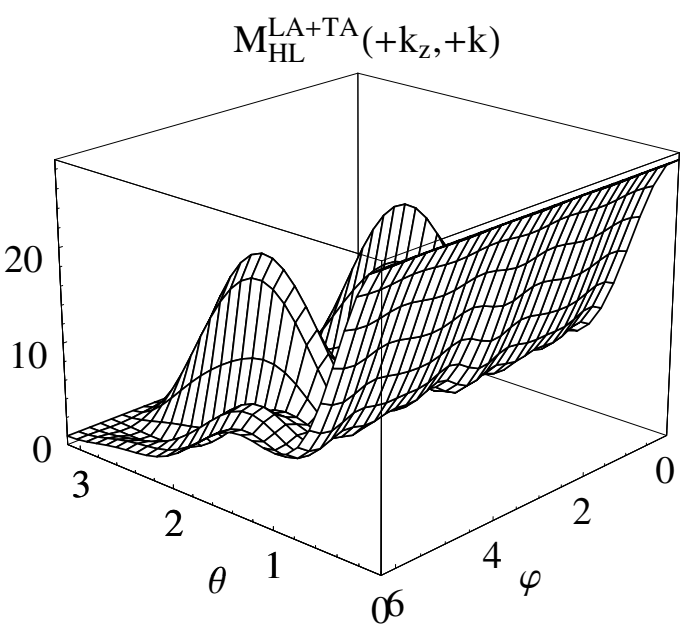

(b)

Fig. 1. Dependence of the square of matrix element for intravalence transitions in the heavy-hole band of GaAs due to sum of LA and TA acoustic phonons. The initial wave vector and spin polarization are parallel to $k_{z}$. The final spin is either (a) aligned or (b) opposed to final wave vector $\mathbf{k}^{\prime}$. $\theta$ and $\varphi$ are the spherical angles of the final wave vector. The square of the matrix elements is in $(\mathrm{eV} / \mathrm{nm})^{2}$ units.

$$
|\mathbf{k}|=\left|\mathbf{k}^{\prime}\right|=0.595 \mathrm{~nm}^{-1} \text {. }
$$

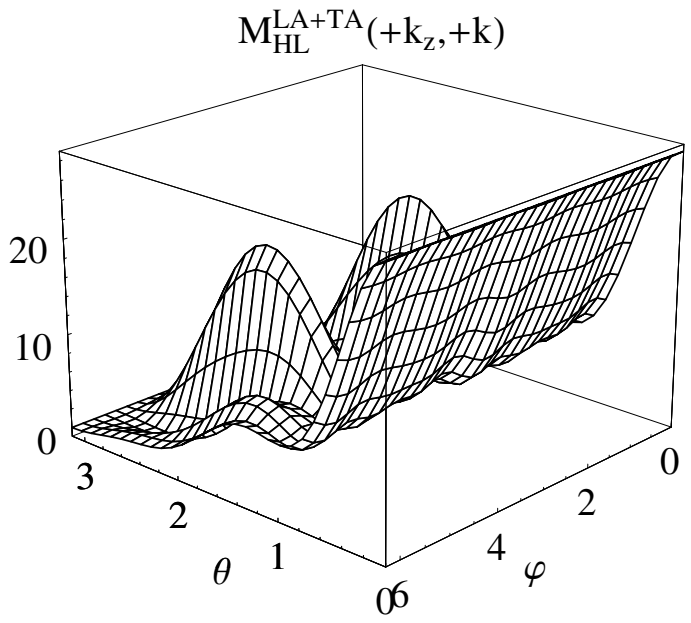

(a)

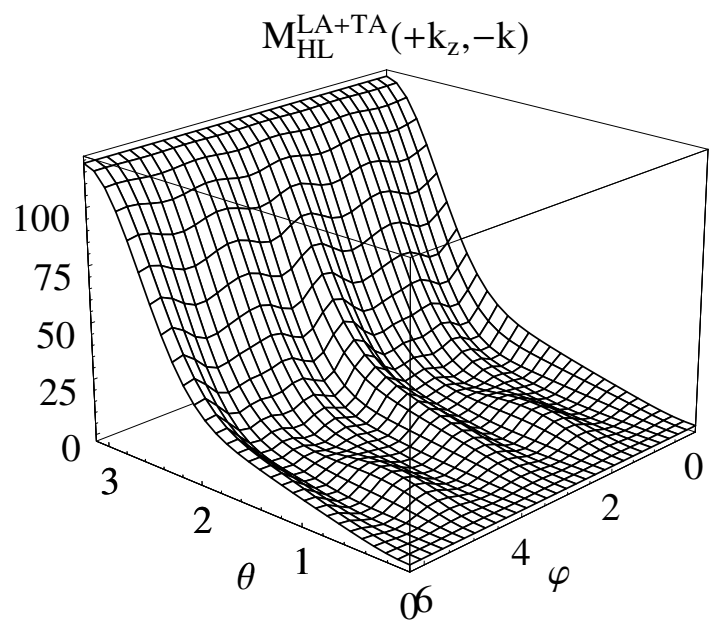

(b)

Fig. 2. The same as in Fig. 1 but for intervalence heavy-light transitions.

opposed to the final wave vector $\mathbf{k}^{\prime}$, for parameters of GaAs and the initial hole wave vector $|\mathbf{k}|=0.92 \mathrm{~nm}^{-1}$ have beeen found:

Intravalence scattering

- $(+k /-k)_{\mathrm{HH}}=0.24$,

- $(+k /-k)_{\mathrm{LL}}=1.15$,

- $(+k /-k)_{\mathrm{SS}}=0.275$.

Intervalence scattering

- $(+k /-k)_{\mathrm{LH}}=0.988$,

- $(+k /-k)_{\mathrm{SH}}=1.33$,

- $(+k /-k)_{\mathrm{SL}}=0.498$.
The calculations have been repeated for other directions of initial and final spin. Nearly in all cases it is found that the "conserving" and "flipping" scattering rates are of comparable magnitudes.

In conclusion, the method to calculate spin scattering matrix elements of holes due to deformation potential interaction for complex valence bands is presented. The method is based on the spin surface concept and is general enough to find matrix elements for various initial and final polarizations of the hole spin. The acoustical and optical deformation potential scattering of the ballistic hole spin, when during collision the hole spin is reversed but the direction of motion does not change, has shown that such spin flipping intraband transitions are forbidden for parabolic as well as 
nonparabolic bands. However, in the case of interband transitions they are allowed. The proposed method allows to connect the spin scattering time with the momentum scattering time. The preliminary calculations show that spin "conserving" and "flipping" transition frequencies are of comparable magnitude and make up half of the momentum scattering frequency at thermal hole energies.

\section{References}

[1] I. Žutić, J. Fabian, and S.D. Sarma, Spintronics: Fundamentals and applications, Rev. Mod. Phys. 76(2), 323-410 (2004).

[2] R.J. Elliott, Theory of the effect of spin-orbit coupling on magnetic resonance in some semiconductors, Phys. Rev. 96(2), 266-279 (1954).

[3] Y. Yafet, $g$ factors and spin-lattice relaxations of conduction electrons, in: Solid State Physics, Vol. 14, eds. F. Seitz and D. Turnbull (New York, Academic Press, 1963) pp. 1-98.

[4] M.I. D'yakonov and V.I. Perel, Spin relaxation of conduction electrons in noncentrosymmetric semiconductors, Sov. Phys. - Solid State 13(12), 3023-3026 (1972). [Fiz. Tverd. Tela 13, 3581 (1971)].

[5] G.L. Bir, A.G. Aronov, and G.E. Pikus, Spin relaxation of electrons due to scattering by holes, Sov. Phys. JETP, 42, 705-712 (1976). [Zh. Eks. Teor. Fiz., 69, 1382 (1975)].

[6] C.P. Slichter, Principles of Magnetic Resonance (Springer, Berlin - Heidelberg - New York, 1980), Chapter 5.

[7] P.H. Song and K.W. Kim, Spin relaxation of conduction electrons in bulk III-V semiconductors, Phys. Rev. B 66(3), 035207-(1-8) (2002).
[8] A.N. Titkov, V.I. Safarov, and G. Lampel, Optical orientation of holes in GaAs, in: Inst. Phys. Conf. Ser. No. 43, 1031-1034 (1979).

[9] B. Baylac, T. Amand, X. Marie, B. Dareys, M. Brousseau, G. Basquet, and V. Thierry-Mieg, Hole spin relaxation in $n$-modulation doped quantum wells, Solid State Commun. 93(1), 57-60 (1995).

[10] D.J. Hilton and C.L. Tang, Optical orientation and femtosecond relaxation of spin-polarized holes in GaAs, Phys. Rev. Lett. 89(14), 146601-(1-4) (2002).

[11] A. Dargys, Coherent properties of hole spin, Lithuanian J. Phys. 43(2), 123-128 (2003).

[12] A. Dargys, Hole spin surfaces in $\mathrm{A}_{3} \mathrm{~B}_{5}$ semiconductors, Phys. Status Solidi B 241(13), 2954-2961 (2004).

[13] A. Dargys, Hole spin dynamics under $\pi$-pulse excitation, Phys. Rev. B 70(12), 125207-(1-11) (2004).

[14] V.F. Gantmakher and Y.B. Levinson, Carrier Scattering in Metals and Semiconductors (North-Holland, Amsterdam, 1987).

[15] M. Tiersten, Acoustic-mode scattering mobility of holes in diamond type semiconductors, J. Phys. Chem. Solids 5, 1151-1168 (1964).

[16] F.L. Madarasz and F. Szmulowicz, Transition rates for acoustic-phonon-hole scattering in $p$-type silicon with nonparabolic bands, Phys. Rev. B 24(8), 4611-4622 (1981).

[17] F. Szmulowicz, Calculation of optical- and acousticphonon-limited conductivity and Hall mobility for $p$ type silicon and germanium, Phys. Rev. B 28(10), 5943-5963 (1983).

[18] G.L. Bir and G.E. Pikus, Symmetry and Strain-Induced Effects in Semiconductors (Wiley, New York, 1974), Chapter 5.

[19] A. Dargys, Hole spin relaxation: Optical deformation potential scattering, Semicond. Sci. Technol. 20(8), 733-739 (2005).

[20] A. Dargys. Unpublished.

\title{
DEFORMACINIO POTENCIALO SAZLYGOTA SUKINIO RELAKSACIJA KUBINIUOSE PUSLAIDININKIUOSE
}

\author{
A. Dargys \\ Puslaidininkiu fizikos institutas, Vilnius, Lietuva
}

\begin{abstract}
Santrauka
Trumpai apžvelgta laisvųjų krūvininku sukinio relaksacija puslaidininkiuose. Aptartas naujas sukinio gyvavimo trukmès skaičiavimo būdas, grindžiamas sukinio paviršiaus koncepcija [11-13]. Sukinio paviršius apibūdina visas įmanomas sukinio poliarizacijas, kai elektronas arba skylè balistiškai juda vienoje iš energinių juostų. Žinant laisvojo krūvininko sukinio paviršių, lengva parinkti spinoriaus parametrus prieš ir po sklaidos, kuriuos būtina žinoti, skaičiuojant su sukiniu susietus matricinius sklaidos elementus.
\end{abstract}

Kiek smulkiau aptarta skylès sukinio relaksacija $p$ tipo puslaidininkiuose, kai skylè sąveikauja su akustiniais ir optiniais fononais per deformacini potencialą. Parodyta, kad dèl stiprios sukinio ir orbitos sąveikos, kuri būdinga elementariuju puslaidininkiu bei III-V, II-VI junginių valentinèms juostoms, skylès sukinio apvertimo tikimybè po susidūrimo su fononu yra didelè. Tai patvirtinta ir eksperimentu. Pateikta sukinio sklaidos matricinių elementų priklausomybė nuo skylès bangos skaičiaus GaAs atveju. 